\title{
AUTOMATIC GENERATION OF ENGLISH LANGUAGE TEST QUESTIONS ON PARTS OF SPEECH
}

\author{
Anna Malinova ${ }^{1}$, Olga Rahneva ${ }^{2}$, Angel Golev ${ }^{3} \S$ \\ ${ }^{1,2,3}$ Faculty of Mathematics and Informatics \\ Plovdiv University "Paisii Hilendarski" \\ 236, Bulgaria Blvd., Plovdiv, 4003, BULGARIA
}

\begin{abstract}
This paper describes computer algebra aided generation of English language test questions for evaluating learners' knowledge of the different parts of speech. The aim of our work is automatic generation of test questions, which are automatically assessed as well. This is a further development of our recent work in the domain; in our previous work we have proposed methods for automatic generation of English language test questions connected with evaluating learners' knowledge of the lexical and grammatical structures that are met in the text; matching words and their meaning; matching parts of the whole; finding synonyms, antonyms and generalizations/specializations of words; test questions for adverbs and adjectives as well as word formation, particularly negative forms of adjectives. As a result, we obtain reduced time consumption during tests authoring, application of equal criteria, fair assessment and decreased influence of subjective factors.
\end{abstract}

Key Words: e-testing, parameterization, dynamic questions, automatic test generation, Wolfram Mathematica, programming

\section{Introduction}

The aim of this work is finding solutions for the automatic generation of En-

Received: $\quad$ September 10, 2016

Revised: December 11, 2016

Published: December 19, 2016

${ }^{\S}$ Correspondence author (c) 2016 Academic Publications, Ltd. url: www.acadpubl.eu 
glish language test questions, which are automatically assessed as well. Test examination is a basic instrument when evaluating various language skills in English. It can be applied at any stage of training - at entry level, for intermediate control and as a final test at the end of the training. A certificate for English language proficiency is required by many institutions in the field of education and business when applying for study or employment [1]. In addition, many countries require adult migrants to demonstrate basic knowledge in the host country language (or at least basic level of English language) before granting residence, work permits or citizenship [2]. A main requirement of test examination is the availability of a large set of test questions in order to avoid cheating or the memorization of answers. Due to the large number of learners and the multiple testing of each of them, the preparation of a sufficient amount of different tests and their assessment is hard and time consuming work. This is a prerequisite for seeking possibilities for intensification of the process of testing and assessment [16].

There are many research efforts in the area of automatic question generation. In [15] is given a literature review of automatic question generation systems which are based on Natural language processing (NLP). The paper presents a review of systems and methodologies developed to automatically generate questions from inputted text. Other extensive researches in the same domain are given in [4], [5], [6], [7] and [18].

However, in our work we apply a different approach parameterization of the test questions as shown below. The aim of the parameterization is obtaining a large number of different but equivalent dynamic questions (with dynamic answers) of a certain topic/subtopic.

A convenient facilities for test authors were developed in DeTC (Distributed e-Testing Cluster) [14], [11], and especially the specialized editor for defining parameterized questions which are automatically assessed. DeTC was initially developed as part of the DeLC (Distributed e-Learning Centre) [17], [3] and later evolved to DisPeL [12], [13] (Distributed Platform for e-Learning), which is now used in several Universities in Bulgaria. In DisPeL has been implemented parameterization of test questions and examination problems in different areas such as: accounting, SQL, electronic circuits, evaluation of the financial efficiency of investment projects, English language and others.

Despite the different approaches and implementation of parameterization caused by the specifics of each of the subject areas, the approach applied in all these cases comes down to the redefinition of a single-answer/multiple-choice question as a dynamic free-type question. The body of the parameterized question contains dynamic variables, whose values vary in a user-defined range. 
Dynamic answers are described with a type, a formula for calculation and a method for comparison with the correct answer. Learners receive unique test questions by automatic generation of the values for the dynamic variables in the parameterized question.

Another tool we have used to automatically generate test questions is the system Wolfram Mathematica [19]. The computer algebra system Wolfram Mathematica is used as a fast and rich programming environment for research work and algorithm creation in the domain.

The following sections discuss the computer algebra aided generation of English language test questions for testing learners' knowledge of different parts of speech.

\section{Previous Work}

In our first results regarding automation of electronic testing examination in the English language [8] we proposed a method for the parameterization of English language test questions of types dichotomy and multiple-choice with one or more correct answers. These are test questions connected with evaluating learners' knowledge of the lexical and grammatical structures that are met in the text. The algorithms were implemented in DisPeL's specialized editor for defining parameterized questions which are automatically assessed.

In [9] we proposed a reimplementation of these results in Wolfram Mathematica in order to use this platform as fast and convenient tool for testing and implementation of new algorithms. The examples in [8] and [9] demonstrate parameterization for dynamically generating a set of questions of type 1 (dichotomy) and type 2 (multiple-choice/single-answer) based on the same input data - values for the dynamic variables and templates for the parameterized sentences. In addition, in [9] we proposed methods for parameterization and automatic generation in Mathematica of English language test questions of the following types:

- Test questions for matching words and their meaning;

- Test questions for matching parts of the whole;

- Test questions for finding synonyms, antonyms and generalizations/specializations of words.

In [10] are described new methods we have developed for the automatic generation of English language test questions in Mathematica. This includes generating: 
- Test questions for testing learner's knowledge of adverbs and adjectives;

- Test questions for word formation, especially negative forms of adjectives.

The results demonstrated that on the basis of a small amount of input values for dynamic variables and patterns for parameterized sentences, we can generate a large set of equivalent dynamic questions of a certain topic/subtopic, chosen by the author.

\section{Automatic Generation of English Language Test Questions for Testing Parts of Speech Using Mathematica}

Parts of speech are the essential building blocks to teaching students English grammar. Understanding the parts of speech and how they work together makes writing and reading easier. There are nine ${ }^{1}$ basic parts of speech and every word will fall into one of these categories. Some words can actually be classified as more than one part of speech depending on the context.

We developed algorithms for automatic generation of English language test questions for testing parts of speech using Wolfram Matheamatica. The Wolfram Language has convenient built-in multilingual dictionaries, built-in information on word meaning, structure, and usage, as well as the relationship between words. Together with Mathematica's tightly integrated string manipulation functions, visualization, and data import and export features, this provides a uniquely powerful platform for natural language computing [19]. For instance, we use Mathematica's functions WordData and TextCases to deal programmatically with the parts of speech. Some examples are given below:

- WordData returns a list of all parts of speech:

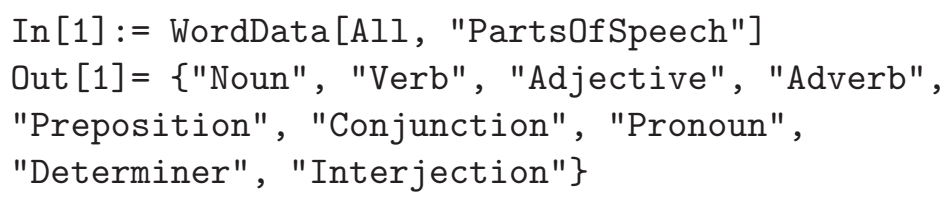

- WordData can give the possible parts of speech for a particular word:

$$
\begin{aligned}
& \text { In }[2]:=\text { WordData["run", "PartsOfSpeech"] } \\
& \text { Out [2] = }\{\text { "Noun", "Verb" }\}
\end{aligned}
$$

\footnotetext{
${ }^{1}$ Some grammar sources traditionally categorize English into 8 parts of speech. Other say 10. In our work, we use the more recent categorization of 9 parts of speech.
} 
- TextCases returns all words of a given part of speech, as it is shown below:

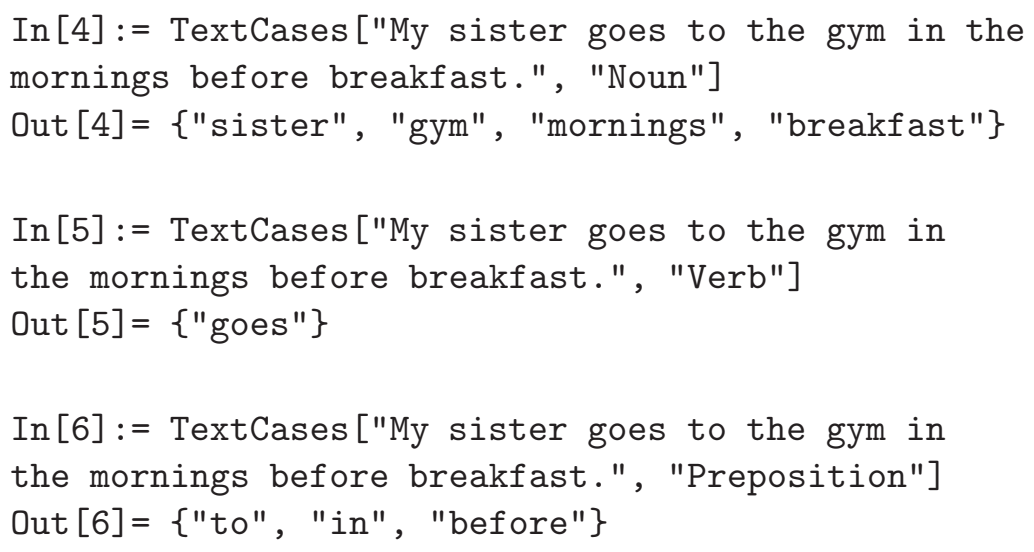

In addition, the function TextStructure in Mathematica generates a nested collection of objects representing the grammatical structure of natural language text. Figure 1 shows text elements tagging parts of speech, while in Figure 2 it is shown the grammatical structure of the sentence "My sister goes to the gym in the mornings before breakfast." (TextStructure generates a tree structure of nested text elements of phrase structure).

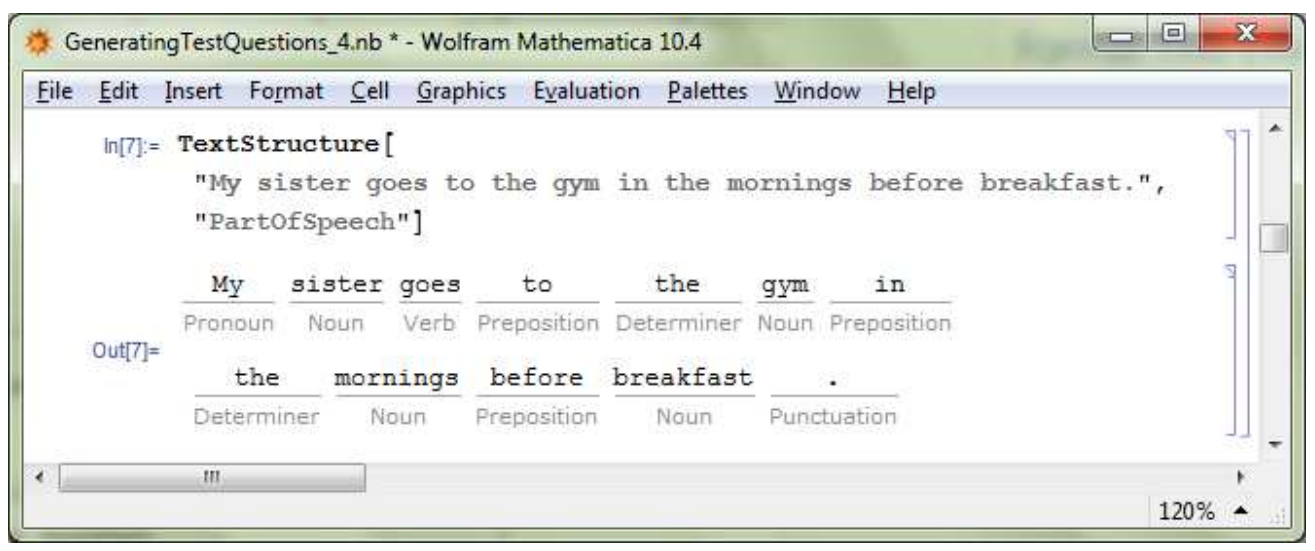

Figure 1: Text elements tagging parts of speech

The algorithm we have implemented in Mathematica for automatic generation of English language test questions for testing the various parts of speech uses the generated in [9] test questions - we use the already generated large number of sentences to create on their basis a large number of test questions for 


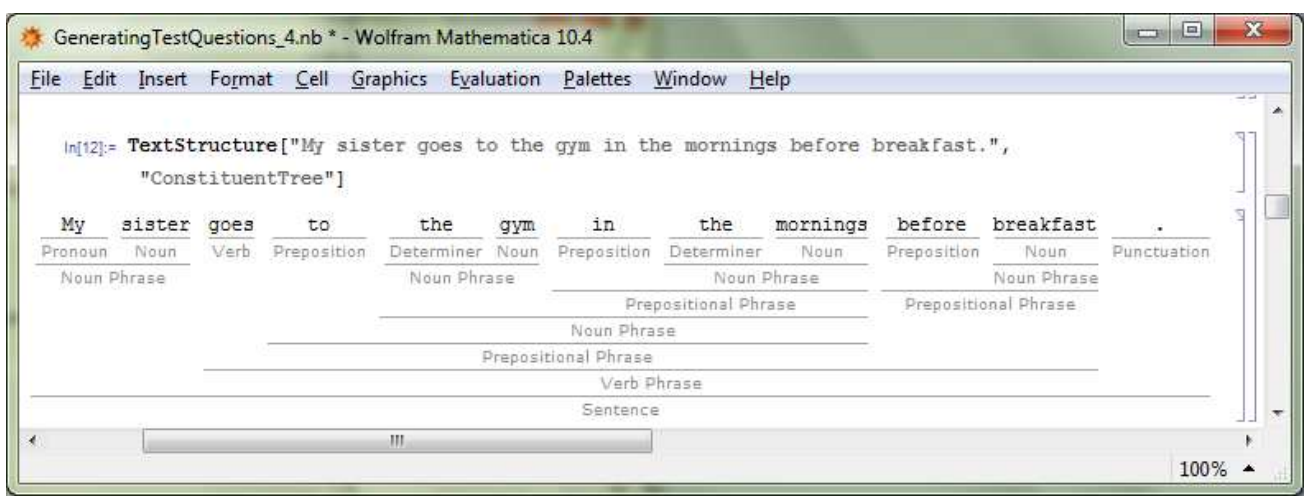

Figure 2: The grammatical structure of a sentence

the parts of speech (see Figure 3 and Figure 4). This way we ensure that each learner receives unique but equivalent test questions of a certain topic/subtopic chosen by the test author. The developed code also uses extensive string manipulation, combinatorics, random number generation, etc.

\section{Conclusion}

The automatic generation of questions allows us to create a large set of equivalent questions of a certain topic based on a small amount of input values. This results in achievements such as reduced time consumption during tests authoring, application of equal criteria, fair assessment and decreased influence of subjective factors.

The computer algebra system Wolfram Mathematica provides significant intensification of the process of English language testing and assessment.

Our future work will be focused on developing further algorithms (and implementing them in DisPeL) for automatic generation of other various English language test questions - questions for determining the underlined option that contains a grammatical or spelling error; questions for adding prepositions; tasks for forming adjectives, nouns, adverbs, plurals; other types of questions for word formation, etc. 


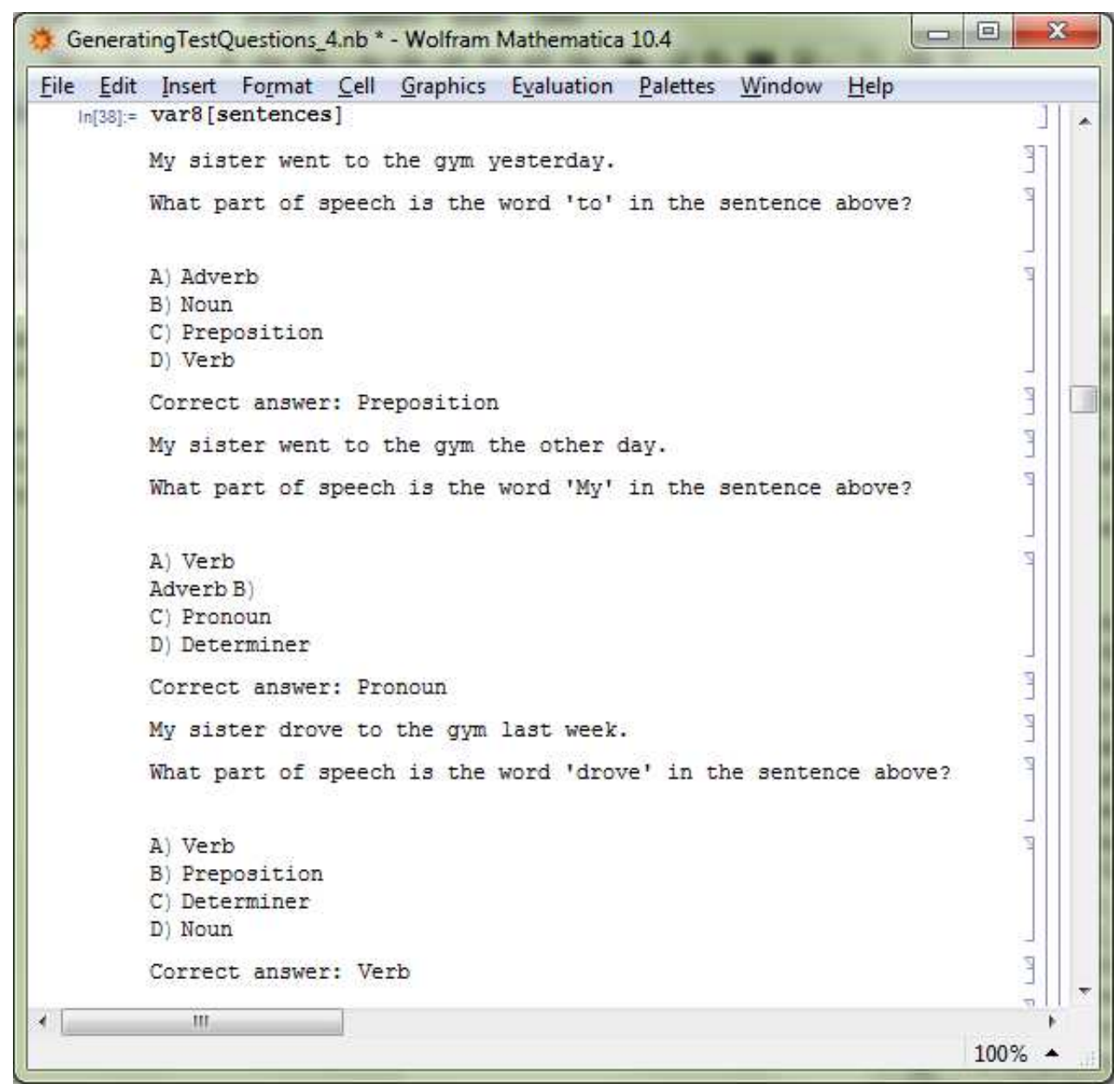

Figure 3: The generated dynamic questions for testing parts of speech (as sentences are used previously automatically generated dynamic questions for evaluating learners' knowledge of the lexical and grammatical structures that are met in the text, Past Simple Tense)

\section{Acknowledgments}

This work is partially supported by the IT15-FMIIT-004 project of the Scientific Fund of the University of Plovdiv "Paisii Hilendarski", Bulgaria. 


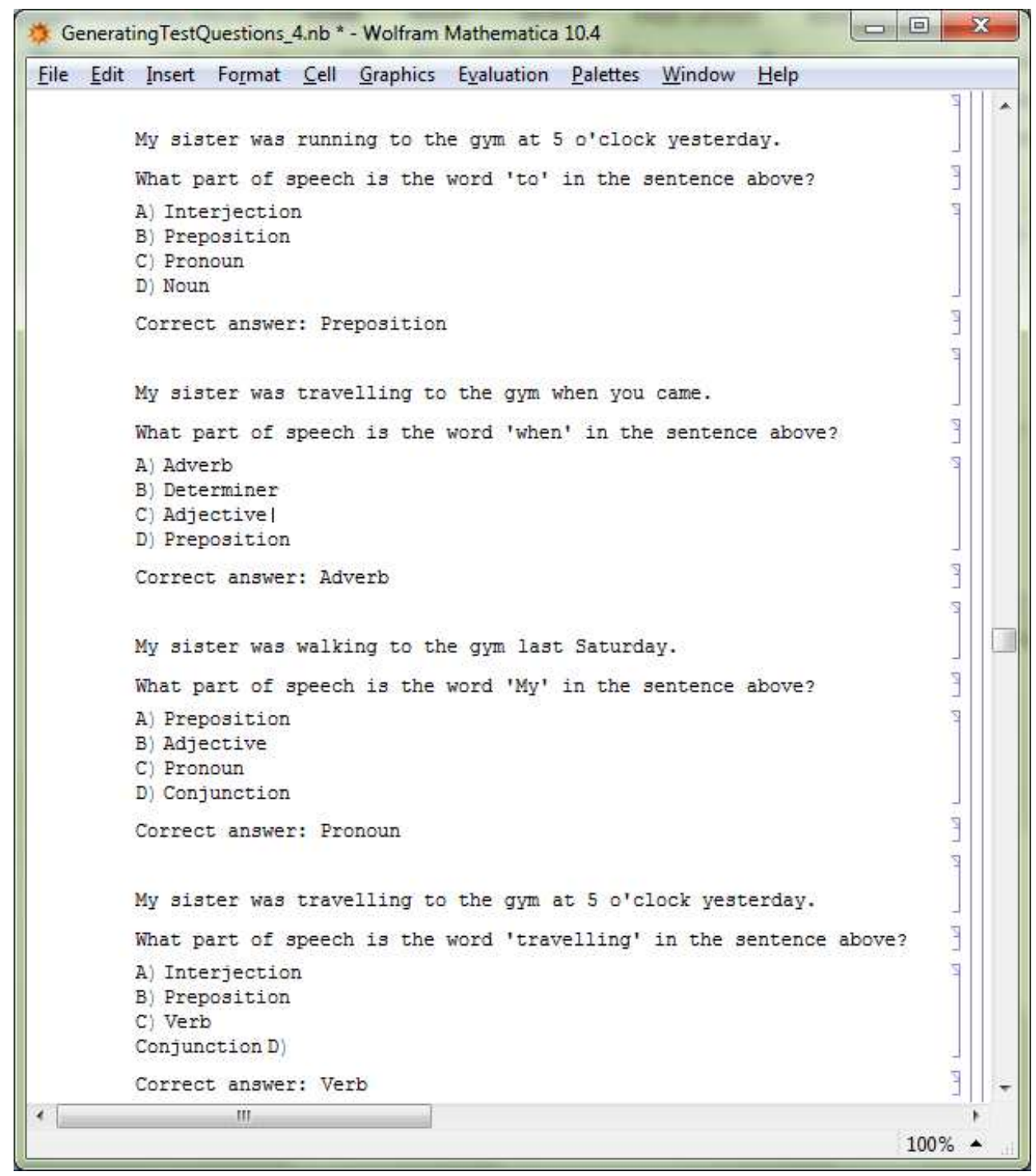

Figure 4: The generated test questions for testing parts of speech (as sentences are used previously automatically generated dynamic questions for evaluating learners' knowledge of the lexical and grammatical structures that are met in the text, Present Continuous Tense) 


\section{References}

[1] COE-2001, The full Common European Framework document of reference for languages: learning, teaching, assessment, Retrived from http://www.coe.int/t/dg4/linguistic/Source/Framework_EN.pdf, last visited on 30.09.2016.

[2] COE-2008, Thematic Studies prepared for the Seminar: "The linguistic integration of adult migrants", Council of Europe, Strasbourg. Retrived from http://www.coe.int/t/dg4/linguistic/Migr_ThematicStudies08_EN.pdf, last visited on 30.09.2016.

[3] Gocheva-Ilieva S., A. Rahnev, New Challenges in e-Learning of Mathematics via EVLM and DeLC Projects, in New Aspects of Engineering Education, Proc. of the 5th WSEAS / IASME Int. Conf. on ENGINEERING EDUCATION (EE'08), Heraklion, Crete Island, Greece, July 22-24, (2008), Published by WSEAS Press, ISBN: 978-960-6766-86-2, ISSN: 1790-2769, (2008), pp. 437-442, invited paper.

[4] Heilman M., Automatic Factual Question Generation from Text, PhD Thesis, Language Technologies Institute, School of Computer Science, Carnegie Mellon University, (2011), http://www.cs.cmu.edu/ark/mheilman/questions/papers/heilman-question-generationdissertation.pdf, last visited on 30.09.2016.

[5] Hoshino A., Nakagawa H., Assisting cloze test making with a web application. In Proceedings of Society for Information Technology and Teacher Education (SITE), San Antonio, Texas, U.S., (2007), pp. 2807-2814.

[6] Hoshino A., Huan L., Nakagawa H., A framework for automatic generation of grammar and vocabulary questions, Proceedings of WorldCALL 2008, Fukuoka, Japan, (2008).

[7] Hoshino A., Huan L., Nakagawa H., Sakumon and sakumon challenge: semiautomated and automated question making systems for grammar and vocabulary testing, Proceedings of WorldCALL 2008, Fukuoka, Japan, (2008).

[8] A. Malinova, V. Ivanova, Automation of Electronic Testing Examination in English Language using DeTC, Plovdiv University "Paisii Hilendarski" Scientific Works, 38 (3Mathematics), (2011), pp. 59-68.

[9] A. Malinova, V. Ivanova, A. Rahnev, Computer algebra aided generation of English language tests, The 11th Annual International Conference on Computer Science and Education in Computer Science CSECS 2015, June 04-07 2015, Boston, MA, USA, Computer Science Education \& Computer Science Research Journal, 11, (2015), pp. 66-74.

[10] A. Malinova, O. Rahneva, Automatic Generation of English Language test Questions Using Mathematica, CBU International Conference "Innovations in Science and Education", Prague, Czech Republic, March 23-25, Vol. 4 (2016): CBU International Conference Proceedings, (2016), pp. 906-909.

[11] Pavlov N., . Rahnev, Architecture and Design of Customer Support System using Microsoft .NET technologies, .NET Technologies 4th International Conference, May 29 June 1 2006, Plzen, Czech Republic, ISBN 80-86943-11-9, (2006), pp. 21-26.

[12] A. Rahnev, N. Pavlov, V. Kyurkchiev, Distributed Platform for e-Learning - DisPeL, European International Journal of Science and Technology (EIJST), 3(1), (2014), pp. 95-109. 
[13] A. Rahnev, N. Pavlov, A. Golev, M. Stieger, T. Gardjeva, New Electronic Education Services Using the Distributed E-Learning Platform (DisPeL), International Electronic Journal of Pure and Applied Mathematics (IEJPAM), 7(2), (2014), pp. 63-71.

[14] Rahneva O., . Rahnev, N. Pavlov, Functional Workflow and Electronic Services In a Distributed Electronic Testing Cluster - DeTC , Proceedings 2nd International Workshop on eServices and eLearning, Otto-von-Guericke Universitaet Magdeburd, 2004, ISBN 3929757-76-1, (2004), pp. 147-157.

[15] Rakangor Sh., Ghodasara, Y., Literature Review of Automatic Question Generation Systems, International Journal of Scientific and Research Publications, 5(1), (2015), pp. $1-5$.

[16] Redecker C., The Use of ICT for the Assessment of Key Competences, European Commission, Joint Research Centre, Publications Office of the European Union, (2013), https://ec.europa.eu/jrc/en/publication/eur-scientific-and-technical-researchreports/use-ict-assessment-key-competences, last visited on 30.09.2016.

[17] Stoyanov S., Ganchev I., Popchev I., ODroma M., Rahnev, A., Glushkova T., Trendafilova M., Inter-University Centre for e-learning and distance learning DeLC, International Scientific Conference Intelligent eLearning Services and Architectures: Problems and Perspectives, Brezovo, 16-17 July 2007, ISBN 978-954-561-303-6, (2007), pp. 17-38.

[18] Susanti Y., Iida R. and Tokunaga T., Automatic Generation of English Vocabulary Tests, Proceedings of the 7th International Conference on Computer Supported Education - Volume 1: CSEDU, ISBN 978-989-758-107-6, (2015), pp. 77-87.

[19] Wolfram Mathematica, http://www.wolfram.com/mathematica/, last visited on 30.09.2016. 